\title{
Unharmed Data Deduplication with Potent Claim Upstairs in Nebula Argosy
}

\author{
Musuluri Abhishek, Surendra Reddy
}

\begin{abstract}
In cloud storage solutions, deduplication era is often accustomed reduce down the location and facts motion goals of offerings with the beneficial resource of the use of casting off repetitive records and moreover storing truly one replica of them. Deduplication is only even as severa clients' supply non-forestall information to the cloud storage; no matter the truth that, it's going to growth issues regarding safety and protection and assets. Evidence of-assets plans allow any form of owner of non-save you information to steer the cloud garage vicinity internet server that he possesses the records ultimately of a sturdy technique. However, severa purchaser's area tool probably to inscribe their facts preceding to outsourcing them to the cloud storage to preserve non-public privateness, however this obstructs deduplication due to the randomisation assets of coding. Recently, numerous deduplication plans are alleged to solve this disadvantage with the resource of the use of permitting every proprietor to percent ordinary coding trick for normal data. However, quite a few the structures be suffering from way of protection problems, at the same time as you take into account that they may be doing no longer take into account the colourful adjustments in the possession of outsourced information that take region ofttimes within the route of an much less expensive cloud storage issuer. For the length of this paper, we have a propensity to advocate a very one-of-a-kind server-trouble deduplication motif for encrypted facts. It lets in the cloud server to govern accessibility to reduced in size out data at the identical time as soon due to the fact the ownership changes dynamically through using exploiting uneven centered coding similarly to relaxed assets cluster important movement. This prevents information discharge not in reality torevokedusers albeit they antecedently had that facts, but in addition to Connect in Nursing honesthowever-curious cloud storage location internet server. Furthermore, the organized trouble count number warranties information honesty in opposition to any shape of tag incongruity assault. Therefore, protection and protection is advanced in the organized motif. The overall performance evaluation effects show display display that the scheduled style is shape of as low-price due to the reality the preceding systems, while the brought remedy fees is minimal.
\end{abstract}

\section{INTRODUCTION}

In cloud agencies, deduplication development is generally made use of to reduce the room and moreover transmission impediment requirements of benefits with the useful resource of butchering bleak facts and furthermore shielding outstanding a solitary replica. Deduplication is brilliant on the identical time as high-quality customers rearrange equal information to the allocated stockpiling, besides it will growth troubles associating with safety further to ownership. Evidence ofownership plans allow any form of proprietor of similar information to show to the appropriated stockpiling server that he insists the statistics in a amazing method.
Regardless, at some problem thing inscribed records is reappropriated right into the cloud restrict and the ownership modifications continuously, deduplication may additionally certainly be obstructed. This manner, we propose a sheltered deduplication tale that enhances dynamic belonging the board primarily based absolutely mostly on randomized simultaneous protection in this examination. When a purchaser uploads expertise that already exist in the cloud storage vicinity, the character need to be hindered from gaining access to the facts which have been hang on preceding to he obtained the ownership via the use of publishing it (backward privateness) 2. These colourful property changes can show up horribly usually in a virtually realistic cloud gadget, similarly to in the end, it ought to be well sorted that lets in you to keep away from the protection and protection degradation of the cloud provider. In the previous technique, most people of the prevailing systems are forecasted a very good way to carry out a prisoner of battle technique in partner degree rate-effective and tough way, because of the fact the hash of the documents, that is handled as a "evidence" for the whole documents, is vulnerable to being leaked to outdoor foes way to its in fact little length. Owner submits statistics that do not exist currently within the cloud garage, he's known as partner diploma initial uploader; if the records exist presently, referred to as a resultant uploader for the reason that this indicates that opportunity residence proprietors ought to possibly have submitted constantknowledge antecedent, he is called a resultant uploader.Severaldeduplication schemes are consider to clear up this downside through the usage of allowing every owner to percent an same cryptography mystery for an identical information. Nonetheless, maximum of the plans be stricken through protection imperfections, for the motive that they will be doing now not undergo in mind the dynamic changes inside the ownership of outsourced data that take location regularly in a very cheap cloud storage area answer. At some component of this paper, we often will be inclined to signify a totally splendid server-aspect deduplication fashion for encrypted statistics. It permits the cloud server to alter accessibility to outsourced information moreover at the same time as the ownership modifications dynamically with the useful useful resource of using exploiting choppy focussed cryptography and additionally comfy belongings collection essential go with the glide. A deduplication fashion over encrypted records. The scheduled trouble depend makes excessive wonderful that excellent prison accessibility to the shared

Revised Manuscript Received on September 10, 2019.

MusuluriAbhishek , CSE department, Newtons Institute of Engineering, AP, India.

Dr.Surendra Reddy, CSE department, Newtons Institute of Engineering, AP, India. 
data is possible, that is considered to be the most essential challenge for fee-powerful further to protected cloud storage offerings within the placing any region assets changes dynamically. It's attained via exploiting a hard and fast a group diverse crucial manage mechanism in every property agency. The deliberate problem don't forget guarantees protection within the setup of prisoner with the beneficial useful usefuluseful resource of introducing a re-encryption gadget that makes use of a in addition collection key for colorful possession collection. Most of the plans are organized to deliver encoding, while however capitalizing a deduplication method, with the beneficial resource of creating it feasible for records domestic proprietors to percent the cryptography keys within the visibility of the inner similarly to outdoor enemies. Given that encrypted statistics square step given to an person.

\section{RELATED WORK}

United protection [1] inscribes an facts paper with the hash estimation of the facts record as an encryption key. Considering that focalized file encryption is deterministic, equal facts are dependably encoded right into same ciphertext, paying little heed to who scrambles them. Thus, the dispensed garage area net server can perform deduplication over the ciphertext, and moreover all owners of the paper can download the ciphertext in addition to unscramble it in some time. United encryption has surely prolonged been centered in company structures and has out of the regular protection variations for comfortable deduplication, which modified into formalized as messagesecured encryption in a while [3] However, they revel in the ill results of protection issues with understand to label uniformity likewise, belongings denial. IDynamic proprietorship modifications in a record sharing event may additionally additionally moreover moreovermoreover show up each now and again in a sensible cloud framework. Regardless, the previous deduplication plans could not entire cozy accumulate control under a colorful property situation. By doing this, for some thing term that refuted customers preserve the encryption thriller, they could accumulate the concerning data inside the allotted storage region any time, this is the problem we corporation to settle on this examination. The cautioned method has the taking place the component of commitments. To begin with, colorful belongings the executives makes exquisite the retrogressive similarly to onward thriller of deduplicated statistics upon any proprietorship alternate. Second, the encouraged plan assurances safety and protection inside the setting of PoW thru supplying a re-encryption tool hat uses a further occasion trick for colorful proprietorship occasion. Subsequently, notwithstanding the truth that that safety secret's exposed, the protection of the re-appropriated records is as however secured in place of outside combatants, at the same time as deduplication stays organized. The fashion of the records deduplication shape consists of the imminent with additives. (1) A information owner encodes the statistics and furthermore redistributes it to the allotted garage space with its list information, that is, a tag; (2) Cloud professional commercial corporation organisation (CSP) deduplicates the redistributed records if essential similarly to stores them. The CSP continues up belongings records for positioned away statistics, which is probably made from a tag for the completed away with information further to the characters of its owners. It manages get proper of get right of entry to toto the positioned away information relying on the proprietorship files similarly to appears after (e.G., problems, refutes, in addition to revitalizes) collect guidelines for each unmarried proprietorship lot as an event.

\section{ENVIRONMENTAL REVIEW \& RESULTS}

It famous the overall communication charges that the cloud net server sustains to deliver out on facts requests from proprietors in a single possession company. Since every organization of files may be seen as an impartial community corporation, we show the simulation consequences adhering to the probabilistic behavior distribution [4] Next, Fig. 2 famous the overall computation fee, sustained at the identical time as an data proprietor secures information in some unspecified time inside the future of upload section among CE [1], LR [2], RCE [3], and the endorsed device. When it come to protection, the proposed device guarantees data privateness, statistics honesty, backward and moreover earlier secrecy, and furthermore collusion eesistance. Thorough application environment, regular overall performance and moreover protection assessment results may be positioned in thefull model of this paper [4] Allow $U=$ fu1; be the universe of customers. Allow IDt be the identity of a client ut.

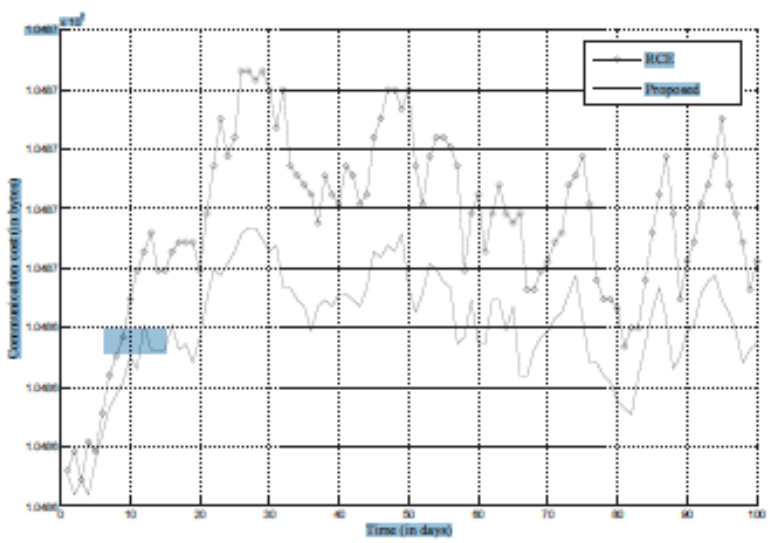

Fig1:Communication cost

Allow $\mathrm{Gi}_{-} \mathrm{U}$ be a hard and rapid of clients that possesses the data $\mathrm{Mi}$. Let $\mathrm{Li}=\langle\mathrm{Ti} ; \mathrm{Gi}\rangle$ be a ownership list for $\mathrm{Mi}$, maintained via the cloud net server, which includes a tag $\mathrm{Ti}$ and $\mathrm{Gi}$ for $\mathrm{Mi}$. Let $\mathrm{KGi}$ be the possession enterprise organisation key this is shared the diverse legitimate owners in Gi. Allow $\operatorname{EK}(\mathrm{M})$ be a symmetrical encryption of a message $\mathrm{M}$ under a key $\mathrm{K}$, and moreover $\mathrm{H}$ : f0; $1 \mathrm{~g} \_$! F0; $1 \mathrm{~g}$ _ be a cryptographic hash characteristic. The encouraged scheme consists of the complying with formula: 1) KEK \$ KEKGen( U): The KEK technology set of policies takes a hard and speedy of customers $U$ as input, and consequences KEKs for every and each customer in U for cozy ownership organization vital distribution. 2) C \$ Encrypt( M; 1 _): The report encryption device isa randomized additives that takes
Blue Eyes Intelligence Engineering

\& Sciences Publication 
as enter facts and moreover a safety parameter _, and outputs a ciphertextC of the data. $\mathrm{C}$ consists of the encrypted message and its tag facts for indexing. Three) $\mathrm{C}, \$$ $\operatorname{ReEncrypt}(\mathrm{C} ; \mathrm{G})$ : The re-encryption method is a randomized set of pointers that takes a ciphertext from its fallen go away to the start place. For ut $2 \mathrm{U}$, PKt denotes a set of the direction keys of ut. When an facts owner ut desires to located up his facts Mi, ut secures the records thru strolling the Encrypt ( $\mathrm{Mi}, 1_{2}$ ) additives, in addition to submits it right into the cloud. Information Re-encryption: Prior to shelling out the ciphertext

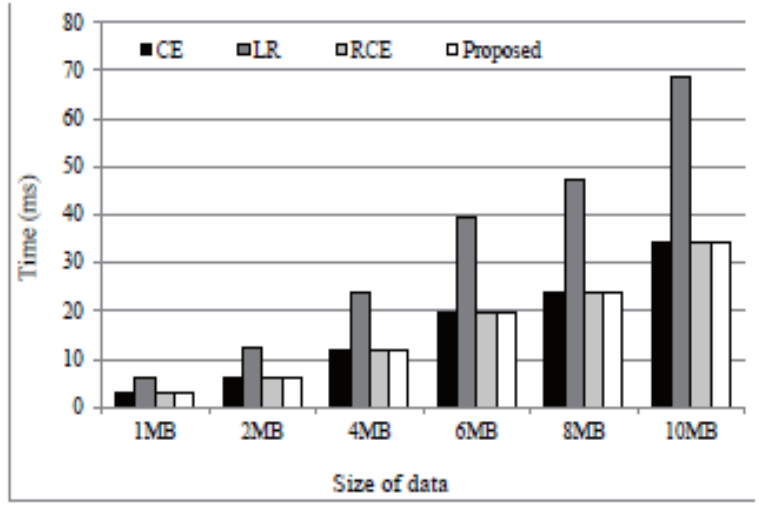

Fig2:Computation time for upload

$\mathrm{Ci}$, the cloud server re-encrypts it with the resource of the use of walking ReEncrypt ( $\mathrm{Ci}, \mathrm{Gi})$ using the ownership business enterprise information for the ciphertext.The reencryption set of regulations applies get proper of get proper of entry to to control of dynamically changing proprietors to the outsourced records. When an individual ut gets a ciphertextC' $i$ from the cloud internet server, he can decrypt the message with the resource of taking walks Decrypt( $C^{\prime}$ $\mathrm{i}, \mathrm{Ki}, \mathrm{PKt}$ ), if ut $2 \mathrm{Gi}$. The information decryptionphase includes ownership organization crucial decryption determined bythe message decryption: In example of next upload, information deletion, and moreover records alteration for $\mathrm{Mi}$, the CSP updates $\mathrm{Gi}$ and furthermore selectsa random possession business enterprise important GK ' $\mathrm{i}(/ \mathrm{GKi})$ and runs theReEncrypt( $\mathrm{Ci}, \mathrm{Gi})$ set of regulations with the upgraded ownershipgroup data $\mathrm{Gi}$ in addition to $\mathrm{GK}$ ' $\mathrm{i}$ as a way to make certain backward/in advance secrecy. Dynamic updates upon any possession modifications in the cloud capability. For this motive, the endorsed technique improvements facts protection in dispersed garage place, at the identical time as permitting whole preferred mind-set to be taken of powerful facts deduplication over scrambled statistics.

\section{CONCLUSION}

We advocated a totally particular comfortable and comfortable records deduplication plan to update notablegrained ownership the pros with the aid of way of abusing the identical antique for the cloud records the board shape. The recommended plot consists of a re-encryption tool that encourages dynamic updates upon any shape of proprietorship changes inside the cloud functionality. Subsequently, the proposed approach enhancements facts safety in dispersed storage vicinity, even as allowing complete preferred problem ofview to be taken of inexperienced information deduplication over scrambled records.

\section{REFERENCES}

1. J. R. Douceur, A. Adya, W. J. Bolosky, D. Simon, as well as M. Theimer," Recovering area from duplicate documents in a serverless distributed filesystem," Proc. International Meeting on Dispersed Computer Equipments( ICDCS), pp. 617-- 624, 2002.

2. J. Xu, E. Chang, as well as J. Zhou, "Leakage-resilient client-side deduplication of encrypted data in cloud storage space," ePrint, IACR http://eprint.iacr.org/2011/538.

3. M. Bellare, S. Keelveedhi, and also T. Ristenpart, "Message-locked security and also safe and secure deduplication," Proc. Eurocrypt 2013, LNCS 7881, pp. 296-- 312, 2013.Cryptology ePrint Archive, Report 2012/631, 2012.

4. J. Hur, D. Koo, Y. Shin, K. Kang, "Secure Data Deduplication with Dynamic Ownership Management in Cloud Storage Space," IEEE Deals on Expertise and Data Engineering, Vol. 28, No. 11, pp. 3113-- 3125,2016. 11.702 . 